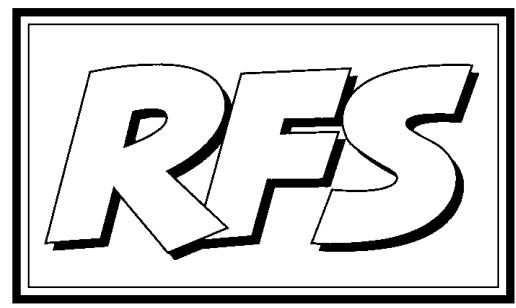

Revista de Fomento Social, 54 (1999), 365-377

\title{
La integración de los países pobres en la economía mundial
}

Incluimos a continuación uno de los textos distribuidos por el Sr. Ricupero con ocasión de su intervención en el seminario «Del riesgo y la vulnerabilidad a la exclusión social: la pobreza en una era de globalización», organizado por la Comisión Pontificia Justicia y Paz en Roma, al que asistió uno de los miembros de nuestro Consejo de Redacción de la Revista de Fomento Social. Este texto fue originariamente presentado en la reunión de «MOST» en París, celebrada el 22 de febrero de 1999 como se puede constatar por varias alusiones temporales del autor. MOST («Management of Social Transformations») es un programa de investigación concebido por la UNESCO para promover la investigación comparativa internacional en el ámbito de las ciencias sociales surgida de la Conferencia de Copenhague. Agradecemos al Sr. Ricupero su autorización para traducir y reproducir aquí el texto. Traducción del original inglés del propio Consejo de Redacción. Los subtitulos son de la RFS.

Rubens RICUPERO (*)

(*) Secretario General de la UNCTAD (Conferencia en las Naciones Unidas sobre Comercio y Desarrollo). 


\section{Preámbulo}

Permítanme que comience expresando mi agradecimiento a mi colega y amigo, el Sr. Mayor Zaragoza, y al secretario y los miembros del comité MOST por haberme invitado tan amablemente. En realidad, no pretendo impartir una conferencia, sino más bien, compartir una serie de reflexiones con Ustedes.

Yohabía siempre tenido muchos deseos de venir a la UNESCO, porque he sido siempre un gran admirador y un gran defensor de su trabajo y también porque en la UNCTAD nosotros compartimos el mismo enfoque básico cultural y humanístico con respecto a las cuestiones económicas y sociales que ha sido siempre la marca de fábrica distintiva de la UNESCO: por eso es para míun motivo de orgullo personal el participar, aunque sea de una forma modesta, en vuestros esfuerzos, y es también una oportunidad para nosotros de aprender de sus preocupaciones en relación con los aspectos sociales del desarrollo económico, lo mismo que en la UNCTAD nosotros nos consagramos a algo más que a lo que se podría describir como «la economía dura» del desarrollo.

Como Ustedes saben, la UNCTAD nació a mediados de los años 60, en una época en que estaban teniendo lugar muchas transformaciones en el mundo, y es un «hijo típico de los 60» en la medida que es el hijo de un pequeño grupo de personas con ideas. Destacan entre ellas un grupo de economistas latinoamericanos, en particular el Dr. RaúlPrebisch, un distinguido economista argentino, Celso Furtado, que vive aquí en París y es una de las personalidades más distinguidas en el desarrollo en América Latina, y el profesor Aníbal Pinto, un distinguido economista chileno que murió hace sólo unos pocos años.

La tarea de la UNCTAD ha sido utilizar las herramientas del comercio y de la inversión para promover el desarrollo y, en particular, el desarrollo de los países menos desarrollados, esos 48 países en los que la gente tiene que vivir con menos de un dólar diario; estos países representan el reto más difícil para la comunidad internacional porque son los miembros más débiles y vulnerables de esa comunidad. Y es exactamente en este momento, en la víspera de un nuevo siglo y de un nuevo milenio, y en medio de una de las crisis más devastadoras para el desarrollo, cuando tenemos que pararnos a reflexionar un poco sobre la experiencia del desarrollo en los últimos 15 o 20 años, e intentar sacar algunas conclusiones. 


\section{La crisis actual}

Voy a intentar describir los actuales esfuerzos para alcanzar algún tipo de consenso emergente sobre la mejor manera de afrontar el desarrollo, evitando al mismo tiempo las trampas de los enfoques unidimensionales que se han seguido durante las últimas décadas. Como siempre, deberíamos empezar partiendo de la propia realidad. Cuando miramos a nuestro alrededor e intentamos fijarnos en la actual crisis económica, que comenzó como una crisis financiera en Asia y que ahora se ha transformado en una crisis de dimensiones mundiales, vemos que afecta a las finanzas, pero que también ha producido efectos devastadores sobre el comercio. Incluso está empezando a tener serias consecuencias sobre las inversiones a largo plazo. Esta crisis, quizás la peor que el mundo haya conocido desde que se estableció el sistema de Bretton Woods en 1944, ya ha provocado una gran cantidad de reflexiones, y se han sugerido diferentes formas de explicar sus causas y sus consecuencias. Independientemente de estas diversas maneras de enfocar el problema, al menos hay dos conclusiones que se desprenden claramente, y que son fácticas e indiscutibles. Una es que la crisis ha afectado a los países en desarrollo mucho más que a los industrializados. La segunda conclusión es que, al contrario de lo que dice la sabiduría convencional, ha tenido más efectos negativos para los países en desarrollo relativamente más avanzados que para los más pobres.

Comencemos por fijarnos en el impacto de la crisis en el mundo desarrollado. De las tres mayores economías industrializadas, Japón ya se encontraba en situación difícil antes de la devaluación tailandesa, desde que estalló la burbuja especulativa de finales de los años 80. Los propios problemas económicos de Japón no fueron una consecuencia de la crisis asiática, aunque existe una interacción entre los problemas económicos de Japón y las perspectivas de desarrollo del Este de Asia. Las otras dos economías industriales del mundo, las de Europa y Estados Unidos, han sido menos afectadas hasta la fecha por la crisis. En alguna medida se han beneficiado de la crisis en razón de los precios extremadamente bajos de las materias primas: en algunos casos, tales como el petróleo o el cobre o el café, la caída de los precios en los últimos 12 meses ha sido de cerca del $40 \%$, hasta un nivel tan bajo que casi no tiene precedentes en los precios de las materias primas. Las economías desarrolladas se han visto también beneficiadas de los menores precios de las manufacturas procedentes de los países que devaluaron sus 
monedas; además atrajeron los flujos financieros que se habían retirado de los países golpeados por la crisis (en lo que se ha dado en llamar «vuelo hacia la calidad»). La economía de los Estados Unidos sigue creciendo y sólo ahora algunas economías europeas que dependen en gran medida de sus exportaciones, como la alemana, han empezado a mostrar algunos signos de estancamiento a causa de la caída de la demanda de importaciones. Sin embargo, en términos generales, la crisis ha sido mucho más negativa para el mundo en desarrollo en general. Esto es cierto respecto de los países de Asia; es verdad ahora en relación con América Latina, que tendrá un muy difícil año 1999; y es también verdad en cuanto a África a causa de la fuerte caída en los precios de las materias primas.

Por primera vez en muchos años, las estimaciones de crecimiento económico de los países en desarrollo durante el presente año son mucho más bajas que las de los países industrializados (menos de la mitad). Por supuesto, ello tendrá serias consecuencias al agravarse más el enorme foso que ya separa las economías ricas de las de los países pobres. La segunda conclusión que hay que sacar de la crisis es que fueron algunos de los países en desarrollo más avanzados los afectados de forma especial por la misma. Ese fue el caso, por ejemplo, de la mayoría de los países asiáticos, donde la crisis se sintió de forma extremadamente fuerte; fue el caso de Tailandia, Malasia, Indonesia, y sobre todo de la República de Corea. En América Latina, Brasil también ha sido ahora muy fuertemente golpeado. Este hecho plantea la siguiente cuestión: ¿cómo se explican Ustedes que países que eran generalmente considerados como verdaderas historias de éxito en términos de desarrollo, hayan podido ser tan vulnerables, incluyendo algunos países que tenían unos fundamentos económicos correctos? Los países asiáticos tenían muy baja inflación y no sufrían déficits presupuestarios. Por el contrario, estos países habían registrado superávits en sus presupuestos públicos durante años. La mayoría tenían altas tasas de ahorro, por lo que no tenían excesiva necesidad de ahorros procedentes del exterior. A pesar de todo eso, fueron afectados de forma extremadamente negativa por la crisis. Siel desarrollo es un proceso que se supone reduce la vulnerabilidad de los países a los choques externos, ¿cómo puede explicarse que algunos de los países que más han avanzado por esa senda, por ejemplo la República de Corea y Malasia, se vieran más afectados que las frágiles economías africanas? ¿No será porque las más avanzadas se encontraban mucho más integradas en la economía mundial? Y, en ese caso, ¿por qué será que un tipo particular de integración habría de aumentar, en vez de disminuir, la vulnerabilidad? 


\section{Más allá del «consenso de Washington»}

De esta forma, tal situación nos obliga a reflexionar sobre el concepto y la experiencia de desarrollo en los últimos años, y a examinar de cerca las recetas o fórmulas que se han propuesto para el desarrollo económico. Evidentemente no estamos ante un problema de desarrollo económico o de pobreza, sin más; el impacto de la crisis es mucho más amplio. En realidad afecta a la economía mundial en general. Suscita cuestiones relacionadas con la liberalización financiera, incluso en los países industrializados. Pero yo me voy a centrar aquí principalmente en las cuestiones relacionadas con el desarrollo económico y con las implicaciones sociales del desarrollo económico, sin perder de vista que existe un vínculo subyacente que conecta estos tres aspectos -desarrollo económico, pobreza y globalización- y que dicho vínculo es el papel y la finalidad de la economía en nuestro tiempo. ¿Cómo debemos abordar la economía? ¿Pertenece realmente la economía al reino de las ciencias exactas? ¿Es la economía algo que tiene leyes, como el sistema planetario, o es un producto de la cultura o el resultado de opciones hechas por la sociedad sobre la base de valores compartidos? He aquí el problema básico que subyace a este debate.

A lo que hoy día estamos asistiendo es a la búsqueda de una alternativa al paradigma de desarrollo que ha sido hegemónico en los últimos 12 años, el así llamado «consenso de Washington». El consenso de Washington recibió este nombre por un artículo de un economista, John Williamson, que intentó codificar el paradigma en una serie de 10 principios. No intentó formularse en 14 puntos, como el Presidente Wilson, con lo que evitó la crítica que hizo Clemenceau a Wilson, diciéndole que incluso el buen Dios se había contentado con sólo diez mandamientos, por lo que debía ser mucho menos ambicioso que el presidente norteamericano. Williamson intentó codificar los diez mandamientos de lo que consideraba «la sabiduría aceptada por todos los economistas serios», como él mismo decía. Su enfoque del desarrollo estaba basado en tres áreas principales. En primer lugar, políticas macroeconómicas sólidas, estoes, baja inflación, déficits públicos mínimos y equilibrios en las cuentas exteriores. En segundo lugar, apertura de los países siguiendo la vía de la liberalización comercial y financiera. Aunque estos dos conceptos usen la misma palabra, liberalización, se trata de diferentes «especies». La liberalización financieraes mucho más difícil de manejar que la liberalización comercial, como ha mostrado la experiencia asiática. Los 
países asiáticos tuvieron muchoéxito en la liberalización comercial, pero no en la liberalización financiera, aunque el consenso de Washington no había distinguido demasiado entre los dos conceptos. Finalmente, el tercer elemento era promover el papel del mercado mucho más que el del Estado, a través de la privatización y la reducción del papel del Estado a las tareas esenciales: desregulación y asuntos similares.

Estas tres áreas han sido básicamente reforzadas por elFMI y el Banco Mundial durante los últimos 12 ó 13 años, en una especie de enfoque «de arriba abajo», un enfoque impuesto mediante las condiciones establecidas para los préstamos del FMI odel Banco Mundial y los principios que inspiraban los llamados programas de ajuste estructural, aplicados durante muchos años en muchos y diferentes países. Estas son las hipótesis que en la actualidad están siendo cuestionadas. Podría decirse que en la actualidad existen tres alternativas principales al consenso de Washington, que noimplican rechazar de plano aquel enfoque, pero sí intentan determinar en qué aspectos el consenso de Washington era inadecuado, dónde debería ser rectificado, o en qué materias deberíamos introducir un balance diferente. Por supuesto, muchos economistas comparten algunas de las premisas implícitas en el consenso de Washington.

Existen básicamente tres alternativas. Una procede del concepto de desarrollo humano, iniciado por el PNUD bajo la guía reciente del Dr. Ul Haq, de Pakistán, con una contribución muy significativa del Profesor Amartya Sen. El concepto de desarrollo humano se fija en aspectos que van mucho más allá de la mera acumulación de capital, del incremento de la productividad económica y del crecimiento de la economía: pone el énfasis en la calidad de vida y presta atención a la educación, a la esperanza de vida y a la calidad del sistema de salud. Es también un enfoque que intenta empezar desde el principio y construir «de abajo arriba», edificando sobre organizaciones basadas en la propia comunidad y en organizaciones no gubernamentales, con muchamayor participaciónigualitaria. Quizás uno de los problemas de este concepto es que resulta todavía relativamente débil en sus fundamentos económicos, es decir, en cómo llegar a tener una base productiva que permita realmente a un país adoptar las políticas sociales que propugna esta manera de entender el desarrollo humano.

La segunda alternativa básica es una que procede del Banco Mundial, el cual se está distanciando progresivamente de la senda más ortodoxa que seguía hasta hace poco tiempo en muchas áreas, tales como su enfoque de los problemas de 
los países altamente endeudados o su reacción a la crisis asiática, cuestiones que son hoy contempladas desde perspectivas diferentes por el FMI y el Banco Mundial. A pesar de todos los intentos diplomáticos por minimizar estas diferencias, nadie puede ocultar el hecho de que el principal economista del Banco Mundial, el profesor Joseph Stiglitz, disiente de forma llamativa de algunas de las premisas básicas del consenso de Washington. Pero ahora el Banco Mundial está yendo más allá de lo que hasta hace poco era considerado como una opinión personal del profesor Stiglitz. Como resultado de las opciones políticas avanzadas por Jim Wolfensohn, presidente del Banco, éste ha anunciado formalmente que va adoptar un nuevo enfoque sobre el desarrollo, el llamado «marco comprehensivo», o «marco estratégico comprehensivo del desarrollo», que el Banco pretende poner en práctica a nivel de cada país. Ya se ha empezado a seleccionar un cierto número de países, uno de los cuales es Bolivia, en los que se aplicará ese «marco comprehensivo». El marco incluye una serie de diferentes aspectos, entre ellos algunos metaeconómicos, tales como cuestiones institucionales, legales y de buen gobierno. Es curioso que muchos de estos elementos están orientados al reforzamiento del Estado, invirtiendo así la tendencia reciente que predicaba una reducción del papel del Estado.

Finalmente, el tercer enfoque, en el que está más directamente implicada la UNCTAD, se sitúa en línea directa con la herencia del pensamiento de Prebisch. Es un intento de combinar el enfoque tradicional latinoamericano de la llamada «escuela histórico-estructuralista», derivada de la Comisión de Naciones Unidas para América Latina y el Caribe (CEPAL), con la experiencia práctica de los países del Este de Asia, intentando adaptar las lecciones de esta experiencia a las economías de África o de los países menos desarrollados. Estas últimas son muy diferentes en su estructura tanto de los países del Este de Asia como de los latinoamericanos, todos ellos países de mediana industrialización que intentan conectar con los países industrializados; en cambio, la mayoría de las economías de África y de los países menos desarrollados son todavía casi exclusivamente economías agrarias, extremadamente dependientes de unas pocas materias primas y con una base productiva muy estrecha. ¿Cómo se pueden desarrollar una serie coherente de enfoques, políticas y medidas que puedan ser relevantes y beneficiosas para estas economías?

Hablando de alternativas, me gustaría insistir en que nadie debería intentar convertir este debate en una nueva versión de las guerras de religión. Nadie niega, 
por ejemplo, que muchas de las cosas del consenso de Washington siguen siendo válidas e imperativas. El mismo profesor Stiglitz tituló una de sus conferencias, «Más allá del consenso de Washington», y todos nosotros estamos precisamente intentando avanzar más allá de él. Acertadamente Stiglitzafirmó en esa conferencia que algunas de las prescripciones del consenso de Washington son necesarias pero no suficientes por sí mismas, y que otras políticas no han merecido la misma atención que estas prescripciones: por ejemplo, él insiste en que la privatización no es siempre una condición necesaria para el desarrollo, y cita el caso de China, el ejemplo más exitoso de crecimiento económico desde 1979, que ha logrado crecimiento sin privatización, a la vez que garantizaba un grado mínimo de competencia en su economía.

\section{Nuevos retos para el desarrollo}

Estamos intentando mirar más allá del consenso de Washington con el fin de alcanzar un consenso común que pueda ser ampliamente compartido, un consenso basado en la necesidad de equilibrio y de un sentido de la proporción, que no vuelva a abrir las viejas batallas ideológicas de los años 70 o de los 80, y que se preocupe, en cambio, de integrar de forma más plena la complejidad y la diversidad de las condiciones que influyen en el desarrollo. En este sentido, queda todavía mucho que hacer para reconciliar extremos aparentemente contradictorios, tales como el papel delEstadoy el mercado, la estabilidad de precios y el crecimiento económico, la flexibilidad del mercado de trabajo y la seguridad en el empleo, la integración en la economía mundial y la construcción de una base industrial nacional. Todos estos aspectos han sido presentados con frecuencia como posiciones antagónicas, mutuamente excluyentes, pero la búsqueda de alternativas que está teniendolugar en la actualidad está inspirada justamente por la necesidad de adoptar un enfoque más multidisciplinary de ver en qué medida podemos alcanzar estos objetivos que se refuerzan mutuamente y son complementarios.

Necesitamos, por tanto, un estudio profundo y comprehensivo de la experiencia del desarrollo durante las últimas décadas, con tres objetivos básicos. El primero es tomar nota de lo que fue acertado y lo que fue equivocado en términos de desarrollo. El segundo es identificar qué faltaba en los enfoques y conceptos originales. Al principio, en los años 50 y 60, el enfoque era excesivamente macroeconómico: ponía especial énfasis en aspectos tales como el crecimiento 
económico, la acumulación de capital y los incrementos de productividad, pero no prestaba la suficiente atención a la calidad del desarrollo, a la calidad de vida y a los aspectos sociales, tales como la distribución de la renta o la distribución de la riqueza. Otros aspectos también totalmente ignorados en los años 60 fueron los relativos a la dimensión medioambiental, la llamada sostenibilidad del desarrollo, el papel de las mujeres en la economía y el papel de las minorías y de las comunidades indígenas. El tercer objetivo del estudio debería ser identificar los retos de futuro: ¿cuál es el reto al que se enfrenta el desarrollo en el siglo próximo, en el próximo milenio?

Alcanzar estos objetivos es una tarea muy difícil, y me abstendré de dar la falsa impresión de que todo el mundo tiene una receta fácil. Creo que nadie puede decir que ya tenemos suficiente conocimiento para resolver las aparentes antinomias del desarrollo. Por poner un solo ejemplo, los economistas no saben todavía lo suficiente sobre cómo conciliar la acumulación de capital con una distribución menos desigual de la renta. Hay economistas que consideran que no existe forma de promover una rápida acumulación de capital sin un incremento simultáneo de la concentración de la renta. Hay todavía muchas cosas que no sabemos, y por eso es tan necesaria la investigación y el enfoque multidisciplinar de las ciencias sociales. Lo necesitamos de la misma forma que necesitamos investigar para encontrar un remedio al SIDA: de alguna manera, se trata del mismo tipo de problemas. La gente suele pensar que una epidemia es de naturaleza diferente al problema del desarrollo, como si en el caso del desarrollo todas las respuestas se conocieran y no hubiera más que aplicarlas para alcanzarlo. Eso no es cierto. Hay todavía muchas cosas que no conocemos acerca del desarrollo económico y social, o de cómo compatibilizar el uno con el otro.

Uno de estos retos mayores es conseguir esa armoniosa complementariedad en un mundo de finanzas globalizadas, donde, como hemos visto recientemente en Asia (en particular, en Indonesia), el progreso alcanzado en los últimos 30 años en la reducción de la pobreza puede irse al traste en cuestión de dos o tres semanas. Se suele tener la tentación de creer que, tan pronto como estas economías puedan recuperarse, sus sociedades recuperarán inmediata y automáticamente el nivel social del que disfrutaban antes de la crisis. Esa no ha sido la experiencia de América Latina, donde todavía hoy, 17 años después del comienzo de la crisis mexicana de la deuda externa, seguida en 1982 por las crisis en Argentina, Brasil,Perú y otros, los indicadores sociales del continente no han retornado al nivel que tenían antes 
de la crisis. El excelente informe de la CEPAL titulado «Panorama social de América Latina», publicado en mayo de 1998, demuestra que el nivel de pobreza en América Latina afecta todavía al $39 \%$ de la población-esto es, que en América Latina hay 209 millones de pobres-, tasa que está 4 puntos por encima del nivel del $35 \%$ anterior a la crisis de 1982. A pesar de tanto sufrimiento y de tan duros esfuerzos, no hemos sido capaces de volver a donde estábamos en 1982. En el caso de algunos países concretos, tales como Chile, los resultados son mejores, pero la CEPAL está hablando de la media del continente como un todo.

Otro ejemplo se refiere a la medición de la indigencia, esto es, los hogares que no son capaces de alimentarse de forma adecuada, aun cuando dediquen toda su renta a alimentación. La indigencia en América Latina alcanza actualmente al 17\% de la población: una de cada seis familias es indigente. Esto significa que la tasa porcentual es dos puntos superior a la que había en 1982. Está claro, por tanto, que no existe una recuperación social automática después de una crisis económica: esto es, que aun cuando el crecimiento económico se recupere, no habrá recuperación automática de los estándares sociales del pasado. Esta experiencia debería ser considerada muy atentamente por los asiáticos, si quieren evitar que se repitan los mismos errores y corregir los mecanismos perversos que desligan el progreso social del crecimiento económico.

\section{El papel del conocimiento y de la información}

Me gustaría hacer hincapié en mis reflexiones conclusivas en uno de los aspectos de este nuevo paradigma emergente que sólo se podrá materializar si trabajamos juntos para hacerlo realidad. Uno de los aspectos de este paradigma tan deseado -que, imagino, será particularmente querido para la UNESCO- es la importancia central del conocimiento y de la información en la economía del futuro. El Profesor Stiglitz, en su carrera académica, ha hecho una contribución importante a esa nueva rama de la economía que los economistas llaman «economía de la información». Esta no se refiere, como mucha gente piensa, a la electrónica o al modo como transmitimos la información a través de las telecomunicaciones. Se refiere a la información en términos económicos. Los economistas clásicos tendían a considerar que la información tenía un coste cero o un coste despreciable: es decir, que cada uno de los agentes presentes en el mercado tenía igual posibilidad de acceso a la información acerca del propio mercado, y de esa forma el coste de adquirir 
información sobre éste podría considerarse nulo o despreciable. La principal contribución de los economistas de la información ha sido demostrar que eso no es verdad; la información tiene un coste, el así llamado «coste de transacción», y a veces este coste de transacción es el que marca la diferencia entre el éxito y el fracaso. Se puede considerar a las empresas como sistemas que negocian con información, y en esto de negociar con información, algunas personas, algunas empresas y algunos países lo hacen mejor que otros. Aquéllos que han tenido una buena educación, una buena formación, tienden a ser mejores en esto de negociar con la información, y suelen tener éxito precisamente allí donde otros fracasan. El problema es qué hacer con los fracasos -esa legión de obreros no cualificados de los países industriales, o los países pobres, incapaces de competir en los mercados porque no tienen el acceso adecuado a la información.

Esto constituye un problema particularmente agudo e importante en nuestros días, por la sencilla razón de que nos movemos hacia un nuevo tipo de economía y hacia una nueva forma de desarrollo, en los que los factores decisivos ya no serán el capital, la mano de obra barata o recursos naturales en abundancia. Cada vez más, el factor central, crucial, es el conocimiento, la información, las patentes; en una palabra, cómo negociar con el conocimiento que se está generando constantemente. A medida que avanzamos haciauna economía intensiva en conocimiento, el acceso a la información y al conocimiento se convierten en lo que marca la diferencia entre prosperidad y pobreza, entre dominación y liberación. Esta es precisamente la razón por la cual la información y el conocimiento habrán de ser cada vez más tenidos en cuenta en las negociaciones en las que se establecen las reglas del juego acerca del comercio y de la inversión, y en todo lo que se refiere a la vida económica en general. Basándome en mi propia experiencia personal durante las negociaciones de la Ronda Uruguay del GATT, les puedo decir que los negociadores comerciales tienden a enfocar el problema de la competencia reduciéndolo a una cuestión de reglas y de arbitrajes. La competencia tiene muchas analogías con un juego y no es accidental que la teoría de juegos, hasta en su formulación matemática, se aplique hoy con frecuencia a la competencia. Como en todojuego, la competencia necesita sin duda que existan y se cumplan las reglas, como las normas de la Organización Mundial del Comercio; y requiere también de un árbitro imparcial, como el mecanismo de solución de diferencias de esa organización. Y los gobiernos y los negociadores comerciales piensan que una vez que tenemos reglas justas y árbitros imparciales, dispondremos ya de las 
condiciones perfectas para la competencia. Pero olvidan por supuesto el tercer y fundamental elemento de la competencia. Para poder jugar un determinadojuego, no basta con conocer las reglas y obedecer al árbitro; además, hay que aprender a jugar; y ello requiere educación y entrenamiento. ¡Nadie puede correr los 100 metros en los Juegos Olímpicos sólo porque haya reglas y árbitros! Entonces, ¿cómo podremos incluir este elemento del aprendizaje y la educación como una parte integrante de la competencia de manera que el juego se desarrolle de forma aceptable? Aunque no podamos asegurar la igualdad en los resultados finales, porque cada jugador posee un nivel de pericia diferente en el juego, al menos tendremos igualdad de oportunidades en el acceso a los entrenamientos para competir. Y esto no es precisamente lo que está ocurriendo.

Permítanme que ponga otro ejemplo relativo al comercio. Federico Mayor ha dicho que «el comercio, no la ayuda» («trade, not aid») debería ser el instrumento del desarrollo, y todo el mundo está de acuerdo con eso. O sea, que ustedes pensarían que la cooperación técnica relacionada con el comercio debería constituir una parte significativa de la cooperación técnica en general. Pues bien, puedo decirles que no es eso lo que ocurre. Las cifras de la OCDE muestran que, de hecho, sólo un 2\% de la cooperación técnica es cooperación relacionada con el comercio. Nadie se ocupa en serio de enseñar a los países cómo producir, cómo comerciar o cómo competir. Esa es la razón por la que la economía de la información debería constituir un elemento importante en la revisión de las reglas relativas al desarrollo, dando por sentado que el «desarrollo» debe entenderse como un proceso de continuo aprendizaje.

En realidad, hemos de admitir que se ha vendido al público en exceso la idea de la globalización a partir de 1989, el año de la caída del muro de Berlín, y en la década que ha seguido desde entonces se ha puesto mucho énfasis en la globalización como un proceso que habría de traer una mayor prosperidad y un crecimiento económico más rápido para todos. De hecho, la primera década de la globalización arroja un resultado descorazonador en términos de crecimiento económico. Esta década ha tenido una de las tasas de crecimiento económico más bajas de los últimos 50 o 60 años -más mediocres aún que la década de los 70 . Por supuesto, cabría decir que la globalización está sólo en sus comienzos. Ahora bien, uno de los problemas de este concepto es que ha sido considerablemente empobrecido al ser presentado casi como sinónimo de integración a escala planetaria de los mercados para el comercio, las finanzas y la inversión directa. Es 
verdad, por supuesto, que globalización es la unificación del espacio económico, pero globalización es mucho más que eso: es un fenómeno cultural e histórico. En las raíces de todo movimiento hacia la globalización, hay siempre una revolución en las ideas, en la ciencia y en la tecnología. Así sucedió al comienzo de la expansión occidental con la revolución galileana en el siglo XVI; asísucedió cuando se produjo la revolución newtoniana en el siglo XVIII, que condujo a la revolución industrial; y así ocurre de nuevo en la actualidad. En esta ocasión la diferencia consiste en que la revolución afecta al tiempo y al espacio, mientras que las anteriores afectaban a la materia y a la energía. Esta vez son los conceptos mismos de distancia y de tiempo los que están siendo modificados por las telecomunicaciones y por la informática, y por eso el problema del acceso a la información se convierte en central. Pero el hecho de que la información, la tecnología y la ciencia sean componentes fundamentales del desarrollo humano no garantiza por símismo que estos elementos no vayan a ser de nuevo utilizados para la opresión o la dominación. En el pasado, el conocimiento científico se usó con demasiada frecuencia para la opresión y la dominación. No deberíamos ser tan ingenuos como para pensar que el acceso a la información es simplemente una cuestión de pedagogía, de aprendizaje, de educación. Hay un elemento de poder - de poder de mercado y de poder político-en el control de la información. De todas formas, la información seguirá siendo la condición crucial para el desarrollo.

Me gusta mucho una frase de Norbert Wiener, el fundador de la cibernética. Solía decir que estar informado es ser libre. Lo que él quería decir es que ser libre es estar capacitado para tomar decisiones, para elegirentre diversas opciones. Pero para poder ser capaz de optar, uno necesita tener el conocimiento necesario acerca de cuáles son las opciones, si existen realmente opciones, y acerca de los costes y beneficios relativos de cada opción, porque en la vida política, lo mismo que en la cultura y en la economía, uno siempre se encuentra ante procesos de «tradeoff» -uno gana algo y pierde algo. Para elegir una opción, uno necesita información. Esperemos que, en esta ocasión, la información servirá, no para oprimir y explotar a los nuevos «dependientes», sino para liberar realmente a la humanidad y promover el verdadero desarrollo humano. 